\title{
Development and Transferability of Black and Red Raspberry Microsatellite Markers from Short-Read Sequences
}

\author{
Michael Dossett ${ }^{2}$ \\ BC Blueberry Council (In partnership with Agriculture and Agri-Food Canada), Pacific Agri-Food \\ Research Centre, P.O. Box 1000, Agassiz, BC, Canada V0M 1 A0 \\ Jill M. Bushakra \\ USDA-ARS, National Clonal Germplasm Repository, 33447 Peoria Road, Corvallis, OR 97333
}

Barbara Gilmore

USDA-ARS, Forage Seed and Cereal Research Unit, 3450 SW Campus Way, Corvallis, OR 97331

Carol A. Koch

Agriculture and Agri-Food Canada, Pacific Agri-Food Research Centre, P.O. Box 1000, Agassiz, BC, Canada VOM $1 A 0$

Chaim Kempler ${ }^{1}$

Agriculture and Agri-Food Canada, Pacific Agri-Food Research Centre, P.O. Box 1000, Agassiz, BC, Canada VOM 1 AO

Chad E. Finn

USDA-ARS, Horticultural Crops Research Unit, 3420 NW Orchard Avenue, Corvallis, OR 97330

\author{
Nahla V. Bassil \\ USDA-ARS, National Clonal Germplasm Repository, 33447 Peoria Road, Corvallis, OR 97333
}

\begin{abstract}
AdDITIONAL INDEX wORDs. Rubus idaeus, Rubus occidentalis, simple sequence repeat, SSR
Abstract. The advent of next-generation, or massively parallel sequencing technologies has been a boon to the cost-effective development of molecular markers, particularly in nonmodel species. Here, we demonstrate the efficiency of microsatellite or simple sequence repeat (SSR) marker development from short-read sequences in black and red raspberry (Rubus occidentalis L. and $R$. idaeus L., respectively), compare transferability of markers across species, and test whether the rate of polymorphism in the recovered markers can be improved upon by how marker sequences are chosen. From 28,536,412 black raspberry reads and 27,430,159 reads in red raspberry, we identified more than 6000 SSR sequences in each species and selected 288 of these (144 from each species), for testing in black and red raspberry. A total of 166 SSR primer pairs were identified with informative polymorphism in one or both species. SSRs selected based on different percentages $(90 \%$ to $\mathbf{9 7 \%}$ as compared with $\geq \mathbf{9 8 \%}$ ) of read cluster similarity did not differ in polymorphism rates from each other or from those originating from singletons. Efficiency of polymorphic SSR recovery was nearly twice as high in black raspberry from black raspberry-derived sequences as from red raspberry-derived sequences, while efficiency of polymorphic SSR recovery in red raspberry was unaffected by the source of the primer sequences. Development of SSR markers that are transferable between red and black raspberry for marker-assisted selection, evaluation of genome collinearity and to facilitate comparative studies in Rubus L. will be more efficient using SSR markers developed from black raspberry sequences.
\end{abstract}

Received for publication 8 Jan. 2015. Accepted for publication 18 Feb. 2015. We would like to thank Mark Dasenko, Caprice Rosato, and Chris Sullivan from the Center for Genome Research and Biocomputing at Oregon State University for their invaluable support in genotyping, sequencing and bioinformatics, respectively. We would also like to thank Liz Alperin and Brian Knauss for their technical support.

This project was supported by funding from the Lower Mainland Horticultural Improvement Association, the Raspberry Industry Development Council of British Columbia, the Washington Red Raspberry Commission, and the Oregon Raspberry and Blackberry Commission, as well as Agriculture and Agri-Food Canada's Growing Forward and Growing Forward 2 Initiatives and USDA-National Institute of Food and Agriculture (NIFA) Specialty Crop Research Initiative (SCRI) Project 2011-51181-30676. Mention of trade names or commercial products in this publication is solely for the purpose of providing scientific information and does not imply recommendation or endorsement by the U.S. Department of Agriculture. ${ }^{1}$ Retired.

${ }^{2}$ Corresponding author. E-mail: Michael.Dossett@agr.gc.ca.
Black raspberry is a relatively minor, but significant, horticultural specialty crop in North America. The majority of black raspberry acreage in North America is in Oregon, where it is grown primarily for processing and had a farm gate value in excess of $\$ 6.8$ million in 2013 [U.S. Department of Agriculture (USDA), 2014]. Smaller, fresh market production acreages are concentrated along the Ohio River Valley and around the Great Lakes, where black raspberry has a long history of cultivation. In addition to being a valuable crop in its own right, black raspberry has also been important in red raspberry breeding, and the majority of red raspberry cultivars developed over the last 30 years have at least some black raspberry ancestry (Hall et al., 2009).

Despite interest in black raspberry for its unique flavor and potential health benefits (Folmer et al., 2014; Kresty et al., 
2001; Seeram, 2008; Stoner et al., 2005, 2008), progress in breeding has been hampered by an apparent lack of genetic diversity in elite germplasm, particularly with regards to insect and disease resistance. Recent efforts to correct this have focused on evaluating wild germplasm and have identified valuable sources of resistance to the aphid Amphorophora agathonica Hottes (Dossett and Finn, 2010), improved vigor and adaptability (Dossett, 2011), and a wealth of genetic diversity not available in black raspberry cultivars (Dossett et al., 2012).

Molecular markers would aid efforts to efficiently integrate traits of interest from wild black raspberry into cultivated germplasm. However, a lack of suitable polymorphic markers in black raspberry is an ongoing problem. The difficulty of identifying useable markers in black raspberry goes back at least 10 years to the first efforts to construct linkage maps in this species (Lewers and Weber, 2005). Dossett et al. (2010, 2012) screened more than 100 SSR primer pairs from red raspberry and blackberry (Rubus subgenus Rubus Watson) to identify 21 polymorphic SSRs in a panel of diverse wild black raspberry accessions. Additional screening efforts of more than 250 SSR markers from a black raspberry expressed sequence tag (EST) library in a mapping population yielded only an additional five polymorphic loci (unpublished data). Recently, Bushakra et al. (2012) published the first linkage map of black raspberry, but they were only able to map 29 polymorphic markers in this species. Although it may be possible to use genotyping by sequencing to generate single nucleotide polymorphism (SNP) markers, or to use amplified fragment length polymorphisms (AFLPs) or other marker systems to develop linkage maps, the development of markers, such as SSRs, that are locus-specific is an ongoing objective in black raspberry for molecular-assisted breeding, transferability of new aphid resistance loci to red raspberry, study of collinearity between the red and black raspberry genomes, understanding fertility issues in crossing these two species, and ultimately to take better advantage of recently published genome sequences in closely related crops, such as strawberry [Fragaria vesca L. (Shulaev et al., 2011)] and peach [Prunus persica L. (Verde et al., 2013)].

The advent of massively parallel, or next-generation, sequencing technology has been a boon to the development of molecular markers and genomic resources for many plant species. Perhaps nowhere has this benefit been realized more than in the study of nonmodel organisms for which research funding is often limited. When this technology first came into use, application toward the discovery of SSR markers was limited to platforms with longer read lengths (e.g., 454 pyrosequencing; Roche, Basel, Switzerland) and was often mined from EST libraries and transcriptome data (Zalapa et al., 2012). Although this remains a popular option for marker development, more recent techniques have been developed to take advantage of high-throughput sequencing of genomic DNA from short read data as short as 80 bp (Gilmore et al., 2013, 2014; Jennings et al., 2011), offering the possibility of obtaining more reads from which to mine data at the expense of read length. This has meant that SSR loci can be identified quickly in mass quantity at a fraction of the time and cost of traditional methods that rely on vector cloning and Sanger sequencing (Zalapa et al., 2012). Despite this, the putative markers must still be tested individually, and the rate of recovery of polymorphic or informative SSRs is often low. The objectives of this study were: 1) to develop genomic SSR markers for use in black and red raspberry from 80-bp pairedend (i.e., short-read) sequencing; 2) to test a subset of 288 dinucleotide and trinucleotide SSR primer pairs developed in black and red raspberry and evaluate polymorphism and transferability between these species; and 3) to determine whether recovery of polymorphic markers from short-read sequences could be improved computationally by selection of SSRs with different levels of read similarity.

\section{Materials and Methods}

Plant materials and DNA isolation. Black and red raspberry parents of mapping populations currently being studied for a variety of different traits were chosen for sequencing. Young, actively growing leaf tissue was collected from plants of the black raspberry selections ORUS 3021-2, ORUS 4153-1, and ORUS 4158-2 at the USDA-Agricultural Research Service (ARS) National Clonal Germplasm Repository in Corvallis, OR. Young, actively growing leaf tissue was also collected from the red raspberry selections and cultivars BC 93-16-43, BC 90-19-34, BC 93-17-11, BC 96-22R-55, 'Chilcotin', and 'Malahat', and sent to the USDA-ARS National Clonal Germplasm Repository in Corvallis, OR for processing. Upon arrival, 30-50 $\mathrm{mg}$ of fresh leaf tissue was placed in a cluster tube (Corning Life Sciences, Tewksbury, $\mathrm{MA})$, frozen in liquid nitrogen, and then stored at $-80{ }^{\circ} \mathrm{C}$ for later DNA extraction using the Gentra Puregene kit (Qiagen, Valencia, CA).

DNA SEQUENCING AND SSR DISCOVERY. DNA sequencing and SSR discovery were similar to the methods described by Gilmore et al. (2014). Briefly, sequencing libraries were prepared from the DNA from each of the black and red raspberry samples described above. Library preparation consisted of: DNA sonication [Bioruptor XL (BR_XL); Diagenode, Denville, $\mathrm{NJ}$; DNA strand end repair; addition of an adenine base to the $3^{\prime}$ ends; ligation of a custom Illumina ${ }^{\circledR}$ sequencing adapter (Illumina, San Diego, CA) containing a 4-bp barcode to the DNA fragments; purification of the ligation products to remove any unligated adapters or adapter-adapter products using a low melting point $1.5 \%$ agarose gel (Lonsa NuSieve, Rockland, $\mathrm{ME}$ ); and excision of an $\approx 300$-bp DNA band from the gel smear with a scalpel (5-Prime SafeXtractor-25; Fisher Scientific, Waltham, MA). The DNA from the excised gel was purified with the QIAquick gel extraction kit (Qiagen) following manufacturer instructions. The purified DNA fragments with adapter molecules on both ends were selectively enriched by polymerase chain reaction (PCR) for 18 cycles. The PCR products were purified using a QIAquick PCR Purification Kit (Qiagen) and then separated on a $1.5 \%$ agarose gel to verify library size and to visually estimate the fragment concentration. The purified library DNA was quantified with the Quant-iT assay (Thermo Fisher Scientific, Carlsbad, CA), using a Qubit fluorometer (Thermo Fisher Scientific), and the 260:280 absorbance ratio was determined as a measure of DNA purity using a ultravioletVis spectrophotometer (NanoDrop ND-1000; Thermo Fisher Scientific). The library size was determined with a Bioanalyzer (Agilent 2100; Agilent Technologies, Wilmington, DE), a chipbased DNA analysis system that separates minute amount of DNA in the presence of an internal standard DNA ladder to generate the quantity and size of each fragment in real time. Each library had a different barcoded adapter to allow assignment of sequences to the correct plant. The black and red 
raspberry sequencing libraries were then diluted and pooled in equimolar amounts by species, and submitted for paired-end 80-bp sequencing in separate lanes by the Genome Analyzer II (Illumina) at the Oregon State University Center for Genome Research and Biocomputing (Corvallis, OR). Illumina ${ }^{\circledR}$ Version 3.0 reagents were used for cluster generation, while sequencing, image acquisition, and base calling were accomplished using the Illumina ${ }^{\circledR}$ pipeline Version 1.5.

Paired-end reads were joined into a single sequence (contig) by concatenating read 1 and the reverse complement of read 2 , separated by $50 \mathrm{Ns}$ to identify the break between reads. The resulting reads were sorted by barcodes using a custom PERL script BCSORT (Knaus, 2011). Sorted reads were searched for di- and trinucleotide motifs. The motif guidelines required that reads contained at least four perfect repeats, i.e., the motif nucleotides were represented in tandem at least four times and had fewer than eight ambiguous $(\mathrm{N})$ bases per sequence. A filter was applied to identify reads with microsatellites located near the center of the sequence. This filter yielded the longest possible flanking sequences for subsequent primer design (Jennings et al., 2011). The program cd-hit-454 (Niu et al., 2010) was then used to filter redundant sequences $(90 \%$ or greater similarity) to a single unique "clustered" read.

Microsatellite MARKer discovery AND SCREening. After read clustering, primers were designed for all of the SSRs using the Genome Database for Rosaceae SSR tool (Jung et al., 2014) with default settings. The resulting primers were sorted to filter out duplicates, compound SSRs, and sequences where either the forward or reverse primer matched other sequences indicating potential paralogs and increased likelihood of amplifying more than one locus. To determine if polymorphism rates could be improved in the markers to be tested by selecting those with slight sequence differences, sequences were then categorized by cluster identity so that SSRs were chosen at random from each of the following categories: $\geq 98 \%$ similarity of clustered reads, $90 \%$ to $97 \%$ similarity of clustered reads, and reads following clustering which still contained only a single sequence (i.e., singletons). A total of 96 dinucleotide SSRs and 48 trinucleotide SSRs from black raspberry and the same number from red raspberry were selected for polymorphism screening. The M13 sequence (TGTAAAACGACGGCCAGT) was added to the $5^{\prime}$ end of each forward primer for economical fluorescent labeling of PCR products as outlined by Schuelke (2000). The forward and reverse primers, along with fluorescently labeled M13 primers (WellRED D2, D3, and D4), were obtained from Integrated DNA Technologies (IDT, San Diego, CA). Fluorescently labeled M13 primers (6-FAM, VIC, NED, and PET) were obtained from Life Technologies (Foster City, CA). Amplification of the M13-tagged SSRs was performed with a touchdown PCR modeled after Schuelke (2000) consisting of an initial denaturing step of $94^{\circ} \mathrm{C}$ for $3 \mathrm{~min}$, then 10 cycles of $94^{\circ} \mathrm{C}$ for $40 \mathrm{~s}, 62^{\circ} \mathrm{C}$ for $45 \mathrm{~s}$ (decreasing the annealing temperature by $1.0^{\circ} \mathrm{C}$ per cycle), and $72{ }^{\circ} \mathrm{C}$ for $45 \mathrm{~s}$ followed by 20 cycles of $94{ }^{\circ} \mathrm{C}$ for $40 \mathrm{~s}, 52^{\circ} \mathrm{C}$ for $45 \mathrm{~s}$, and $72{ }^{\circ} \mathrm{C}$ for $45 \mathrm{~s}$; eight cycles of $94^{\circ} \mathrm{C}$ for $40 \mathrm{~s}, 53^{\circ} \mathrm{C}$ for $45 \mathrm{~s}$, and $72^{\circ} \mathrm{C}$ for $45 \mathrm{~s}$; and a final extension of $72^{\circ} \mathrm{C}$ for $30 \mathrm{~min}$.

In Corvallis, the black and red raspberry primer sets were screened in the black raspberry selections ORUS 3021-2, ORUS 4153-1, and ORUS 4158-2, as well as a subset of progeny from the crosses ORUS 3021-2 × ORUS 4153-1 and ORUS 4158-2 $\times$ ORUS 3021-2 to determine allele polymorphism and segregation via capillary electrophoresis (CE) with the Beckman CEQ 8000 capillary genetic analyzer (Beckman Coulter, Fullerton, CA). In Agassiz, the same primer sets were screened in the red raspberries 'Malahat', BC 93-16-43, and $\mathrm{BC}$ 90-19-34 as well as a subset of seedlings from the crosses BC 93-16-43 × 'Malahat' and 'Malahat' $\times$ BC 90-19-34 to determine allele polymorphism and segregation via $\mathrm{CE}$ using the Applied Biosystems 3730S 48-capillary DNA Analyzer (Life Technologies) at the University of British Columbia NAPS Unit (Vancouver, BC, Canada). As a check for consistency of protocols and because of differences in equipment at each location, the black raspberry ORUS 4304-77 (from the cross of ORUS 4158-2 $\times$ ORUS 3021-2) was amplified in Agassiz for comparison with the Corvallis results. To further reduce analysis cost, PCR products at both locations were pooled into multiplexes consisting of one sample of each fluorescent tag for a total of three (black raspberry) or four (red raspberry) samples per multiplex before CE. Peaks were called by fitting into bins that were less than one nucleotide wide using the fragment analysis module of the CEQ 8000 (Beckman Coulter) in Corvallis, and Peak Scanner v2.0 (Life Technologies) in Agassiz and followed by manual confirmation of size of each peak. PCR reactions from primers failing to amplify products were repeated before classification. PCR amplifications for SSR primers that resulted in either excessive stutter or an excessive degree of nonspecific priming resulting in electropherograms that were difficult to interpret were classified as noninformative and excluded from the results, while those primer pairs which resulted in fragments that could be easily discerned with size differences segregating in the study populations were classified as having informative polymorphism. Finally, results for PCR amplification of each primer pair in red and black raspberry were combined to compare rates of informative polymorphism and transferability with previous work as well as to identify those which were useable in both the red raspberry and black raspberry mapping populations.

COMPARISON OF POLYMORPHISM RATES. Comparisons of informative polymorphism rates between methods of choosing read clusters was made using Chi-square tests (expected ratio $1: 1: 1)$ within each combination of repeat type, species of origin and species of test, as well as Student's $t$ test (paired, twotailed) across categories of repeat type, species of origin, and species of test for each combination of $\geq 98 \%$ similarity of clustered reads, $90 \%$ to $97 \%$ similarity of clustered reads, and singletons. Comparison of transferability rates based on species of origin was made using Student's $t$ test (unpaired, two-tailed) while assessment of species-of-origin bias for markers polymorphic in both species was made using Chi-square tests comparing observed rates of polymorphism in both species to expected rates calculated from the conditional probabilities of polymorphism in both species. All statistics were performed with SAS (version 9.1; SAS Institute, Cary, NC).

\section{Results}

Sequencing results, as measured by rates of SSR marker recovery from clustered reads were similar across species but slightly higher for black raspberry than for red raspberry. A total of 28,536,412 reads were generated from sequencing in black raspberry, while 27,430,159 reads were generated from sequencing in red raspberry. From these, 6234 dinucleotide and 449 trinucleotide repeats were recovered from black raspberry sequences while a total of 5837 dinucleotide and 
378 trinucleotide repeats were recovered from red raspberry sequences (Table 1).

A high proportion of the SSR primers tested amplified products. In black raspberry, 124 of $144(86 \%)$ SSRs from red raspberry-derived sequences amplified while 137 of 144 (95\%) SSRs from black raspberry-derived sequences amplified products. In red raspberry, 118 of 144 (82\%) SSRs from black raspberry-derived sequences amplified products while 130 of $144(90 \%)$ SSRs from red raspberry-derived sequences amplified products. Of the 144 primer pairs from each species, a total of 87 black raspberry and 78 red raspberry primer pairs yielded informative polymorphic products (Table 2). Tests comparing the three methods of choosing SSRs from read clusters were not significant for all comparisons within groups of repeat type, species of origin, and species of test (range of $\chi^{2}=0.21-3.27$, $P=0.19-0.88$ ). Similarly, all tests across these groupings were also not significant $(t=0.65,0.64$, and $1.49, P=0.53,0.54$ and 0.18 , for $\geq 98 \%$ read similarity vs. $90 \%$ to $97 \%, 90 \%$ to $97 \%$ read similarity vs. singletons, and $\geq 98 \%$ read similarity vs. singletons, respectively) indicating that the rate of informative polymorphic SSRs was unaffected by selecting SSRs from any of the three categories of read clusters.

In black raspberry, almost twice as many informative SSRs were recovered when they were developed from black raspberry-derived sequences (64) compared with those developed from red raspberry sequences $[34, t=10.29, P \leq 0.001$ (Tables 2 and 3)]. Interestingly, this same result did not hold true for markers in red raspberry. In red raspberry, a similar number of polymorphic SSRs were recovered from red raspberry-derived sequences (55) as from black raspberry-derived sequences [ 49 , $t=1.40, P=0.23$ (Tables 2 and 3)]. There were more SSRs with informative polymorphisms in both species when they were derived from black raspberry sequences [25 SSRs (17\%)] than when they were derived from red raspberry sequences [12 SSRs $(8 \%)]$, but this was because there were twice as many SSRs from black raspberry sequences that were polymorphic in black raspberry to start with; the numbers reflect the conditional probabilities of polymorphism in both species without additional bias toward one species or the other $\left(\chi^{2}=0.32, P=0.57\right.$ and $\chi^{2}=0.43, P=0.51$, for markers developed from black and red raspberry sequences, respectively). Summary data for the polymorphic markers can be found in Table 3.

\section{Discussion}

Massively parallel sequencing technologies have significantly reduced the cost of development of SSR primer pairs by eliminating the need for enriched libraries, vector cloning, and Sanger sequencing (Zalapa et al., 2012). Further reductions in cost can be made by barcoding and multiplexing different individuals and/or species during sequencing (Jennings et al., 2011), use of primers with M13 adapters (Schuelke, 2000), and multiplexing PCR products before CE. The ability to increase the rate of informative polymorphism by prescreening SSR markers in-silico, thereby reducing the amount of time and resources spent screening uninformative markers, would be another cost-cutting step. Iorizzo et al. (2011) illustrated this potential by mining SSRs from transcriptome data of carrots from four different genetic backgrounds. They identified 114 SSRs that were computationally polymorphic and confirmed that 26 of 31 tested $(84 \%)$ were actually polymorphic. This compares to an average SSR polymorphism rate of $29 \%$ in SSRs developed from 454 pyrosequencing in a survey by Zalapa et al. (2012).

Table 1. Recovery statistics for sequencing reads and SSR-containing sequences and clusters from black and red raspberry.

\begin{tabular}{lcccccc}
\hline & & \multicolumn{2}{c}{ Dinucleotide repeats } & & \multicolumn{2}{c}{ Trinucleotide repeats } \\
\cline { 3 - 7 } & Reads (no.) & $\begin{array}{c}\text { SSR-containing } \\
\text { sequences (no.) }\end{array}$ & $\begin{array}{c}\text { SSR-containing sequences } \\
\text { after clustering (no.) }\end{array}$ & & $\begin{array}{c}\text { SSR-containing } \\
\text { sequences (no.) }\end{array}$ & $\begin{array}{c}\text { SSR-containing sequences } \\
\text { after clustering (no.) }\end{array}$ \\
\hline Black raspberry & $28,536,412$ & 7,921 & 6,234 & 516 & 449 \\
Red raspberry & $27,430,159$ & 7,856 & 5,837 & 515 & 378 \\
\hline
\end{tabular}

Table 2. Summary of SSRs developed from 80-bp paired-end short-read sequences of black and red raspberry with informative polymorphisms. The same primer pairs were tested in both species. All comparisons of polymorphism rates are statistically nonsignificant except the rate of polymorphic primer pairs in black raspberry, which was significantly higher in primer pairs derived from black raspberry sequences as compared with those derived from red raspberry sequences $(P \leq 0.001)$.

\begin{tabular}{|c|c|c|c|c|}
\hline & \multicolumn{2}{|c|}{ Dinucleotide repeats } & \multicolumn{2}{|c|}{ Trinucleotide repeats } \\
\hline & $\begin{array}{c}\text { Developed from } \\
\text { black raspberry (no.) }\end{array}$ & $\begin{array}{l}\text { Developed from } \\
\text { red raspberry (no.) }\end{array}$ & $\begin{array}{c}\text { Developed from } \\
\text { black raspberry (no.) }\end{array}$ & $\begin{array}{l}\text { Developed from } \\
\text { red raspberry (no.) }\end{array}$ \\
\hline \multicolumn{5}{|l|}{ Black raspberry } \\
\hline $90 \%$ to $97 \%$ read similarity & $16(32)$ & $13(47)$ & $2(4)$ & $2(6)$ \\
\hline Singletons & $20(48)$ & $3(13)$ & $13(35)$ & $7(34)$ \\
\hline Total & $45(96)$ & $24(96)$ & $19(48)$ & $10(48)$ \\
\hline$\geq 98 \%$ read similarity & $2(16)$ & $10(36)$ & $1(9)$ & $4(8)$ \\
\hline $90 \%$ to $97 \%$ read similarity & $13(32)$ & $14(47)$ & $0(4)$ & $2(6)$ \\
\hline Singletons & $19(48)$ & $5(13)$ & $14(35)$ & $20(34)$ \\
\hline Total & $34(96)$ & $29(96)$ & $15(48)$ & $26(48)$ \\
\hline Polymorphic in both species & $19(96)$ & $9(96)$ & $6(48)$ & $3(48)$ \\
\hline
\end{tabular}

${ }^{\mathrm{z}}$ The number of SSRs attempted in each class is indicated in parentheses. 


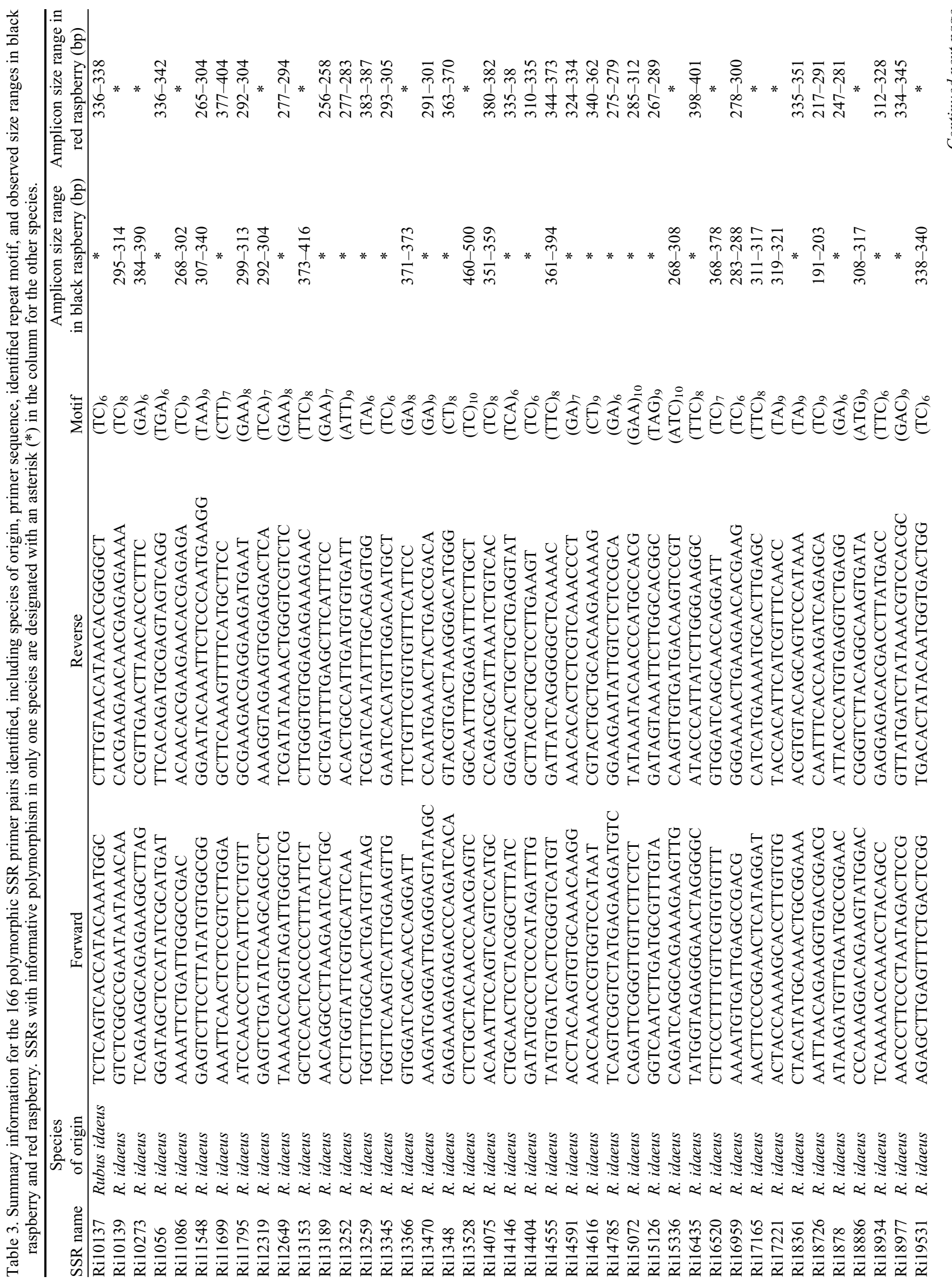




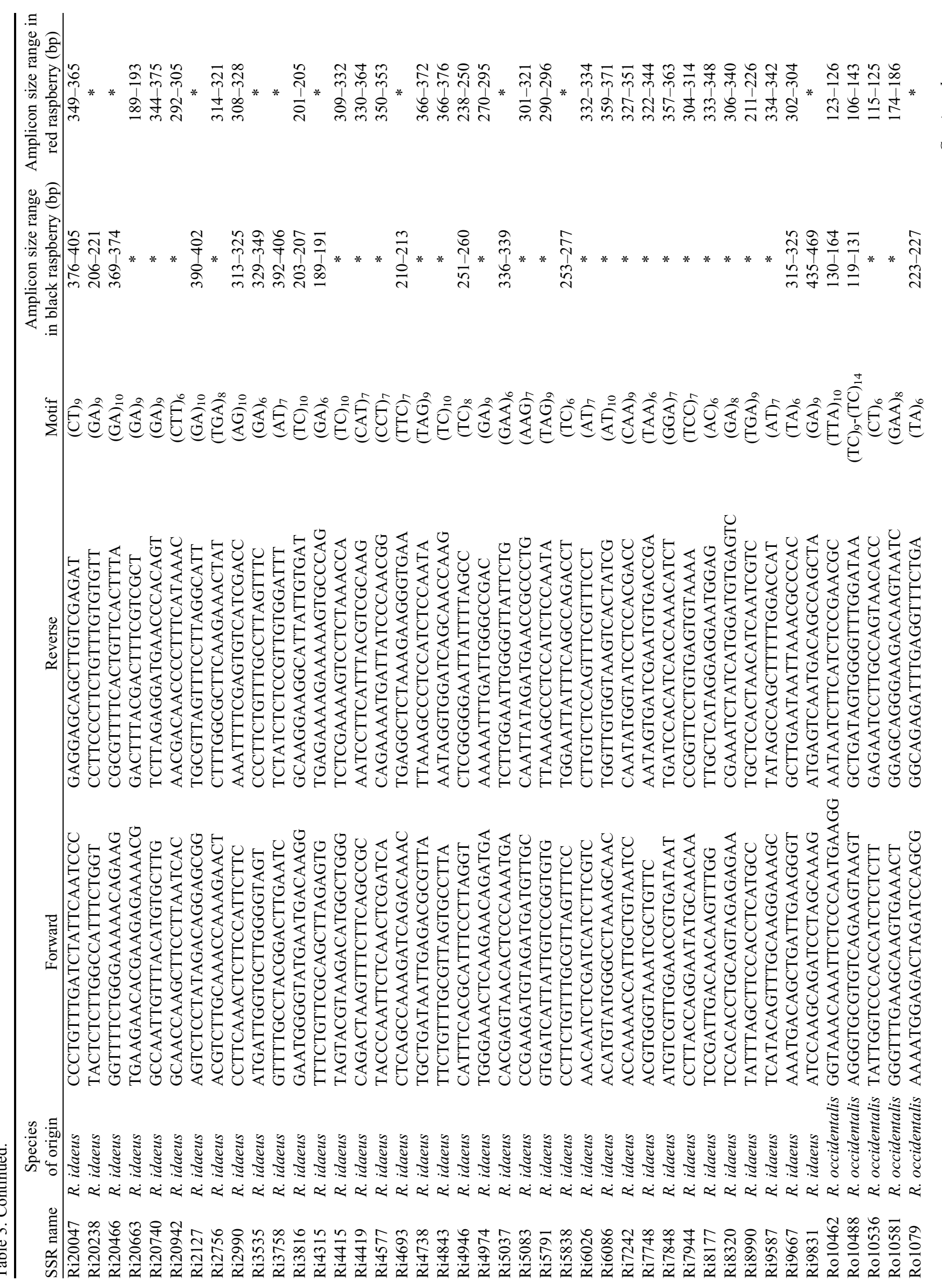




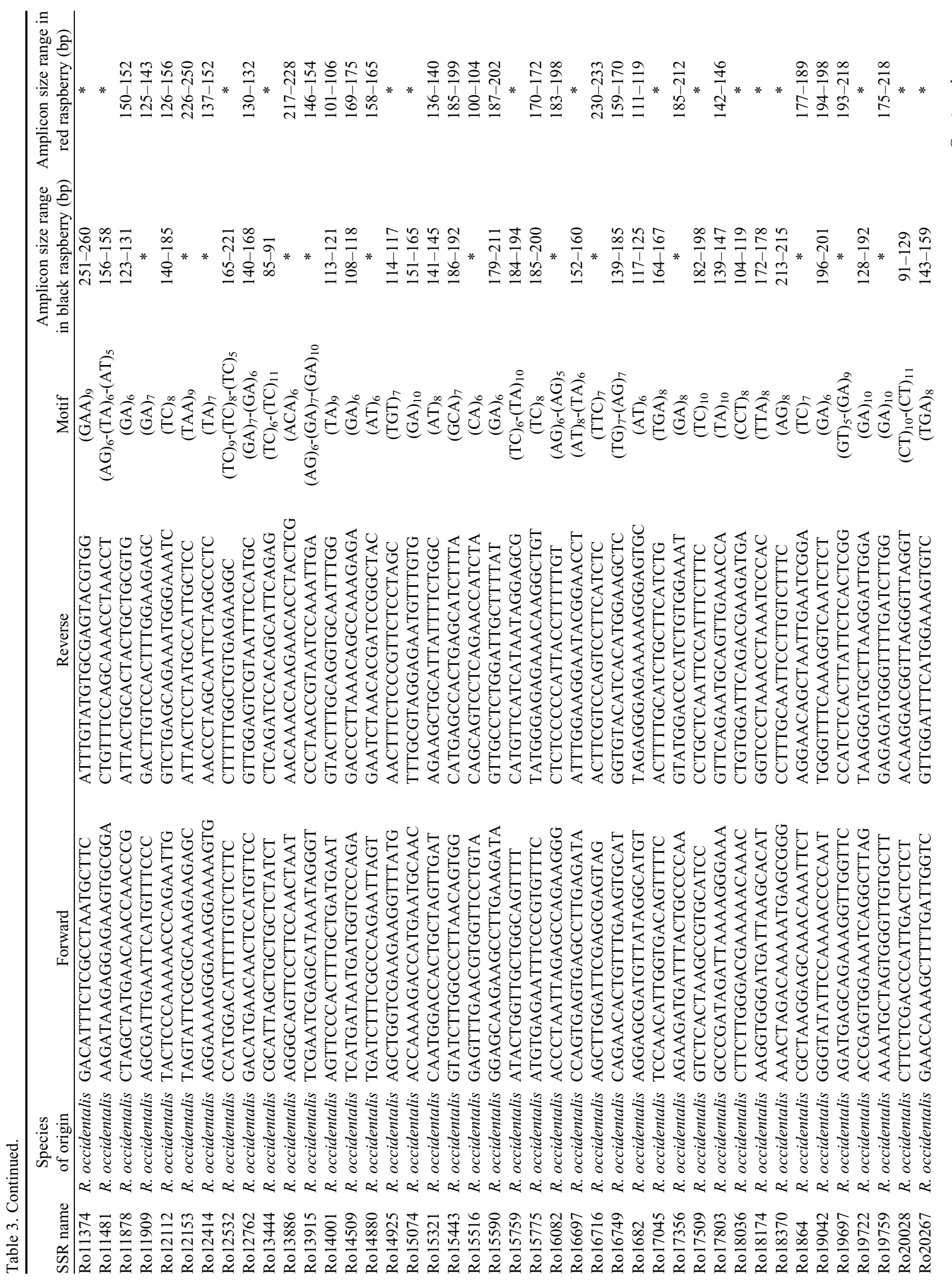




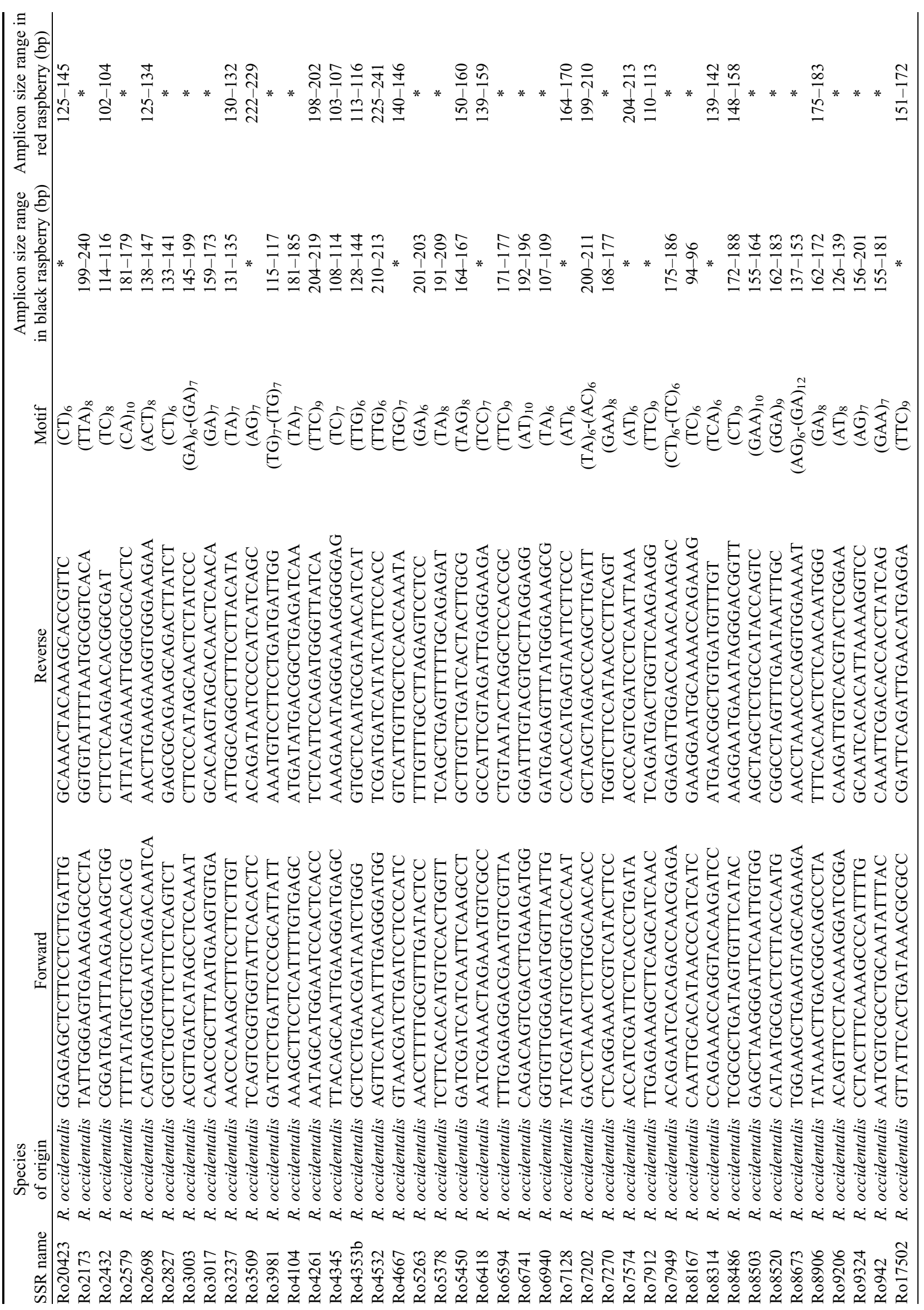


We attempted to see if we could achieve similar success in increasing polymorphism rates by choosing SSRs from sequences with high similarity but which were not identical. We were unable to show recovery of higher rates of polymorphic markers by identifying clusters with slight sequence differences. Although filtering read clusters from sequences by degree of sequence similarity may seem like a possible strategy for quickly identifying potentially polymorphic sequences, this did not turn out to be the case. In retrospect, this is likely because sequences had already been filtered to identify those sequences in which the repeat motif was located near the center of the sequence (i.e., abutting the $50 \mathrm{Ns}$ placed in the middle of the paired-end sequence). This was done to identify SSR sequences with the maximum available flanking sequence for primer design, but may have resulted in an inability to determine the exact number of repeats in each motif, thereby limiting the ability to computationally determine fragment size polymorphisms. Similarly, this also limits the ability to distinguish between simple and compound SSRs. Because of a sorting error which was not discovered until after primers had been ordered, several of the black raspberry dinucleotide repeats that were chosen had compound motifs (Table 3). This is unlikely to have substantively affected the results because many of the other SSRs tested likely also have compound motifs that were obscured by the missing data at the center of the paired-end sequences. Although this and other recent studies have shown that it is quite possible to develop large numbers of SSR markers de novo at relatively low cost from short-read sequences, longer reads are still desirable for many applications and are an area of rapid improvement as the technology matures. More recent versions of the Illumina ${ }^{\circledR}$ technology, or other sequencing platforms featuring longer read lengths might mitigate these limitations in the future.

In this study, the average rate of polymorphism ranged from $21 \%$ to $54 \%$ (Table 2) depending on the repeat type and source. Specifically, the rates of transferability of markers from red raspberry that are also polymorphic in black raspberry were similar to previous work. Dossett et al. (2010) found about $20 \%$ of EST- and genomic-SSR markers from red raspberry and a slightly lower number of blackberry EST-SSR markers were polymorphic in a panel of 16 cultivated and wild black raspberry accessions. We might expect somewhat higher rates of polymorphism if evaluating these SSRs in a broader set of germplasm, but this is unlikely to substantively change the results. Of $21 \mathrm{SSR}$ initially identified as polymorphic in a panel of 16 wild accessions and then used to study black raspberry diversity by Dossett et al. (2012), 19 (90\%) were polymorphic in one or both of the parents and progeny representing the two black raspberry mapping populations evaluated here (unpublished data). Likewise, the parents of the two red raspberry mapping populations that were evaluated are from diverse backgrounds, with 'Malahat' having mixed but primarily European red raspberry ancestry (Kempler and Daubeny, 2000) while BC 90-19-34 and BC 93-16-43 are $\mathrm{F}_{1}$ hybrids with wild North American Rubus strigosus Michx. (Dossett et al., 2013). Furthermore, an evaluation of SSRs from other red raspberry linkage maps (Graham et al., 2004, 2006; Woodhead et al., 2008, 2010) has shown that $86 \%$ of the markers tested to date are polymorphic in one or both of these mapping populations (unpublished data).

\section{Conclusions}

This study adds to the growing body of work demonstrating that new SSR markers can be efficiently and economically developed from next-generation sequencing of genomic DNA using a combination of barcoded samples and massively parallel sequencing technology, even with short read lengths. In this study, we identified 166 new polymorphic SSR primer pairs from 288 tested in black and red raspberry. Of these, 37 were polymorphic in both species. Our method of attempting to increase the rate of polymorphism by selecting SSRs from clustered reads with $90 \%$ to $97 \%$ similarity did not have any benefit over choosing SSRs from clustered reads of higher similarity or from singletons.

The rate of successful SSR development in black raspberry was nearly twice as high from sequences originating from black raspberry as compared with red raspberry, while success in red raspberry was about the same, regardless of whether the markers came from black or red raspberry sequences. Because of this, genomic raspberry-derived SSR markers will be of the most utility for both species if they are developed from black raspberry sequences.

\section{Literature Cited}

Bushakra, J.M., M.J. Stephens, A.N. Atmadjaja, K.S. Lewers, V.V. Symonds, J.A. Udall, D. Chagné, E.J. Buck, and S.E. Gardiner. 2012. Construction of black (Rubus occidentalis) and red (R. idaeus) raspberry linkage maps and their comparison to the genomes of strawberry, apple, and peach. Theor. Appl. Genet. 125:311-327.

Dossett, M. 2011. Evaluation of genetic diversity in wild populations of black raspberry (Rubus occidentalis L.). PhD Diss., Oregon State Univ., Corvallis.

Dossett, M. and C.E. Finn. 2010. Identification of resistance to the large raspberry aphid in black raspberry. J. Amer. Soc. Hort. Sci. 125:438-444.

Dossett, M., N. Bassil, and C.E. Finn. 2010. Transferability of Rubus microsatellite markers to black raspberry. Acta Hort. 859:103-110.

Dossett, M., N.V. Bassil, K.S. Lewers, and C.E. Finn. 2012. Genetic diversity in wild and cultivated black raspberry (Rubus occidentalis L.) evaluated by simple sequence repeat markers. Genet. Resources Crop Evol. 2012:1849-1865.

Dossett, M., C. Kempler, and H. Daubeny. 2013. BC 90-19-34 and BC 93-16-43 red raspberries. HortScience 48:664-667.

Folmer, F., U. Basavaraju, M. Jaspars, G. Hold, E. El-Omar, M. Dicato, and M. Diedrich. 2014. Anticancer effects of bioactive berry compounds. Phytochem. Rev. 13:295-322.

Gilmore, B., N. Bassil, A. Nyberg, B. Knaus, D.L. Barney, and K. Hummer. 2013. Microsatellite marker development in peony using next generation sequencing. J. Amer. Soc. Hort. Sci. 138:64-74.

Gilmore, B., N.V. Bassil, D.L. Barney, B.J. Knaus, and K.E. Hummer. 2014. Short-read DNA sequencing yields microsatellite markers for Rheum. J. Amer. Soc. Hort. Sci. 139:22-29.

Graham, J., K. Smith, K. MacKenzie, L. Jorgenson, C. Hackett, and W. Powell. 2004. The construction of a genetic linkage map of red raspberry (Rubus idaeus subsp. idaeus) based on AFLPs, genomicSSR and EST-SSR markers. Theor. Appl. Genet. 109:704-749.

Graham, J., K. Smith, I. Tierney, K. MacKenzie, and C.A. Hackett. 2006. Mapping gene $H$ controlling cane pubescence in raspberry and its association with resistance to cane botrytis and spur blight, rust and cane spot. Theor. Appl. Genet. 112:818-831.

Hall, H.K., K.E. Hummer, A.R. Jamieson, S.N. Jennings, and C.A. Weber. 2009. Raspberry breeding and genetics. Plant Breed. Rev. 32:39-382.

Iorizzo, M., D.A. Senalik, D. Grzebelus, M. Bowman, P.F. Cavagnaro, M. Matvienko, H. Ashrafi, A. Van Deynze, and P.W. Simon. 2011. De novo assembly and characterization of the carrot transcriptome reveals novel genes, new markers, and genetic diversity. BMC Genomics 12:389.

Jennings, T.N., B.J. Knaus, T.D. Mullins, S.M. Haig, and R.C. Cronn. 2011. Multiplexed microsatellite recovery using massively parallel sequencing. Mol. Ecol. Resources 11:1060-1067. 
Jung, S., S.P. Ficklin, T. Lee, C. Cheng, A. Blenda, P. Zheng, J. Yu, A. Bonbarely, I. Cho, S. Ru, K. Evans, C. Peace, A.G. Abbott, L.A. Mueller, M.A. Olmstead, and D. Main. 2014. The genome database for Rosaceae (GDR): Year 10 update. Nucleic Acids Res. 42:D1237-D1244.

Kempler, C. and H.A. Daubeny. 2000. 'Malahat' red raspberry. HortScience 35:783-785.

Knaus, B.J. 2011. Short read toolbox. 21 Nov. 2014. $<$ http://brianknaus. com/software/srtoolbox/>.

Kresty, L.A., M.A. Morse, C. Morgan, P.S. Carlton, J. Lu, A. Gupta, M. Blackwood, and G.D. Stoner. 2001. Chemoprevention of esophageal tumorigenesis by dietary administration of lyophilized black raspberries. Cancer Res. 61:6112-6119.

Lewers, K.S. and C.A. Weber. 2005. The trouble with genetic mapping of raspberry. HortScience 40:1108. (Abstr.).

Niu, B., L. Fu, S. Sun, and W. Li. 2010. Artificial and natural duplicates in pyrosequencing reads of metagenomic data. BMC Bioinformatics 11:187.

Schuelke, M. 2000. An economic method for the fluorescent labeling of PCR fragments. Nat. Biotechnol. 18:233-234.

Seeram, N.P. 2008. Berry fruits: Compositional elements, biochemical activities, and the impact of their intake on human health, performance, and disease. J. Agr. Food Chem. 56:627-629.

Shulaev, V., D.J. Sargent, R.N. Crowhurst, T.C. Mockler, O. Folkerts, A.L. Delcher, P. Jaiswal, K. Mockaitis, A. Liston, S.P. Mane, P. Burns, T.M. Davis, J.P. Slovin, N. Bassil, R.P. Hellens, C. Evans, T. Harkins, C. Kodira, B. Desany, O.R. Crasta, R.V. Jensen, A.C. Allan, T.P. Michael, J.C. Setubal, J. Celton, D.J.G. Rees, K.P. Williams, S.H. Holt, J.J.R. Rojas, M. Chatterjee, B. Liu, H. Silva, L. Meisel, A. Adato, S.A. Filichkin, M. Troggio, R. Viola, T. Ashman, H. Wang, P. Dharmawardhana, J. Elser, R. Raja, H.D. Priest, D.W. Bryant, Jr., S.E. Fox, S.A. Givan, L.J. Wilhelm, S. Naithani, A. Christoffels, D.Y. Salma, J. Carter, E.L. Girona, A. Zdepski, W. Wang, R.A. Kerstetter, W. Schwab, S.S. Korban, J. Davik, A. Monfort, B. DenoyesRothan, P. Arus, R. Mittler, B. Flinn, A. Aharoni, J.L. Bennetzen, S.L. Salzberg, A.W. Dickerman, R. Velasco, M. Borodovsky, R.E. Veilleux, and K.M. Folta. 2011. The genome of the woodland strawberry (Fragaria vesca). Nat. Genet. 43:109-118.
Stoner, G.D., C. Sardo, G. Apseloff, D. Mullet, W. Wargo, V. Pound, A. Singh, J. Sanders, R. Aziz, B. Casto, and X.L. Sunday. 2005. Pharmacokinetics of anthocyanins and ellagic acid in healthy volunteers fed freeze-dried black raspberries daily for 7 days. J. Clin. Pharmacol. 45:1153-1164.

Stoner, G.D., L.S. Wang, and B.C. Casto. 2008. Laboratory and clinical studies of cancer chemoprevention by antioxidants in berries. Carcinogenesis 29:1665-1674.

U.S. Department of Agriculture. 2014. National statistics for raspberries. 3 Dec. 2014. <http://www.nass.usda.gov/Statistics_by_Subject/result. php?8884AB14-2056-36CD-92F2-550B9DBACC94\&sector $=$ CROPS\& group=FRUIT $\% 20 \% 26 \% 20$ TREE $\% 20$ NUTS\&comm $=$ RASPBERRIES $>$.

Verde, I., A.G. Abbott, S. Scalabrin, S. Jung, S. Shu, F. Marroni, T. Zhebentyayeva, M.T. Dettori, J. Grimwood, F. Cattonaro, A. Zuccolo, L. Rossini, J. Jenkins, E. Vendramin, L.A. Meisel, V. Decroocq, B. Sosinski, S. Prochnik, T. Mitros, A. Policriti, G. Cipriani, L. Dondini, S. Ficklin, D.M. Goostein, P. Xuan, C. Del Fabbro, V. Aramini, D. Copetti, S. Gonzalez, D.S. Horner, R. Falchi, S. Lucas, E. Mica, J. Maldonado, B. Lazzari, D. Bielenberg, R. Pirona, M. Miculan, A. Barakat, R. Testolin, A. Stella, S. Tartarini, P. Tonutti, P. Arús, A. Orellana, C. Wells, D. Main, G. Vizzotto, H. Silva, F. Salamini, J. Schmutz, M. Morgante, and D.S. Rokhsar. 2013. The high-quality draft genome of peach (Prunus persica) identifies unique patterns of genetic diversity, domestication and genome evolution. Nat. Genet. 45:487-494.

Woodhead, M., S. McCallum, K. Smith, L. Cardle, L. Mazzitelli, and J. Graham. 2008. Identification, characterisation and mapping of simple sequence repeat (SSR) markers from raspberry root and bud ESTs. Mol. Breed. 22:555-563.

Woodhead, M., A. Weir, K. Smith, S. McCallum, K. MacKenzie, and J. Graham. 2010. Functional markers for red raspberry. J. Amer. Soc. Hort. Sci. 135:418-427.

Zalapa, J.E., H. Cuevas, H. Zhu, S. Stefan, D. Senalik, E. Zeldin, B. McCown, R. Harbut, and P. Simon. 2012. Using next generation sequencing approaches to isolate simple sequence repeat (SSR) loci in the plant sciences. Amer. J. Bot. 99:193208. 